\title{
Association Between Cadmium Exposure and Liver Function in Adults in the United States: A Cross-sectional Study
}

\author{
Dongui Hong ${ }^{1,2}$, Jin-Young Min ${ }^{3}$, Kyoung-Bok Min ${ }^{1,2}$ \\ ${ }^{1}$ Department of Preventive Medicine, Seoul National University College of Medicine, Seoul, Korea; ${ }^{2}$ Integrated Major in Innovative Medical Science, \\ Seoul National University Graduate School, Seoul, Korea; ${ }^{3}$ Veterans Medical Research Institute, Veterans Health Service Medical Center, Seoul, Korea
}

Objectives: Cadmium is widely used, leading to extensive environmental and occupational exposure. Unlike other organs, for which the harmful and carcinogenic effects of cadmium have been established, the hepatotoxicity of cadmium remains unclear. Some studies detected correlations between cadmium exposure and hepatotoxicity, but others concluded that they were not associated. Thus, we investigated the relationship between cadmium and liver damage in the general population.

Methods: In total, 11838 adult participants from National Health and Nutrition Examination Survey 1999-2015 were included. Urinary cadmium levels and the following liver function parameters were measured: alanine aminotransferase (ALT), aspartate aminotransferase (AST), gamma glutamyl transferase (GGT), total bilirubin (TB), and alkaline phosphatase (ALP). Linear and logistic regression analyses were performed to assess the associations between urinary cadmium concentrations and each liver function parameter after adjusting for age, sex, race/ethnicity, annual family income, smoking status, alcohol consumption status, physical activity, and body mass index.

Results: The covariate-adjusted results of the linear regression analyses showed significant positive relationships between log-transformed urinary cadmium levels and each log-transformed liver function parameter, where beta \pm standard error of ALT, AST, GGT, TB, and ALP were $0.049 \pm 0.008(p<0.001), 0.030 \pm 0.006(p<0.001), 0.093 \pm 0.011(p<0.001), 0.034 \pm 0.009(p<0.001)$, and $0.040 \pm 0.005$ $(p<0.001)$, respectively. Logistic regression also revealed statistically significant results. The odds ratios ( $95 \%$ confidence intervals) of elevated ALT, AST, GGT, TB, and ALP per unit increase in log-transformed urinary cadmium concentration were 1.360 (1.210 to 1.528), 1.307 (1.149 to 1.486 ), 1.520 (1.357 to 1.704 ), 1.201 (1.003 to 1.438 ), and 1.568 (1.277 to 1.926 ), respectively.

Conclusions: Chronic exposure to cadmium showed positive associations with liver damage.

Key words: Cadmium, Liver, Heavy metals, Alanine transaminase, Aspartate aminotransferases

Received: August 7, 2021 Accepted: November 5, 2021

Corresponding author: Kyoung-Bok Min

Department of Preventive Medicine, Seoul National University College of Medicine, 103 Daehak-ro, Jongno-gu, Seoul 03080, Korea

E-mail: minkb@snu.ac.kr

This is an Open Access article distributed under the terms of the Creative Commons Attribution Non-Commercial License (https://creativecommons.org/licenses/bync/4.0// which permits unrestricted non-commercial use, distribution, and reproduction in any medium, provided the original work is properly cited.

\section{INTRODUCTION}

Cadmium is a toxic heavy metal that is commonly used as a corrosion-resistant material. However, in the human body, it is non-essential and non-biodegradable [1]. Occupational exposure to cadmium can occur during nickel-cadmium battery manufacturing, zinc mining, and cadmium welding. Apart from occupational exposure, environmental exposure to cadmium 
can also occur through smoking cigarettes and consuming contaminated water or food [2]. Cadmium is absorbed into the human body predominantly via the respiratory tract and secondarily via the gastrointestinal tract [2,3]. Urinary excretion accounts for most of the elimination of cadmium, although some of it may also be excreted through stool, saliva, and milk [1-3]. However, as the excretion rate of cadmium is low, its biological half-life is extremely long (approximately 20-30 years in humans) [4]. The long half-life of cadmium can maximize its damage to target organs, such as the kidney and liver [4]. The effect of cadmium on the kidney is well documented; however, relatively few studies have focused on the liver.

The majority of published studies on the association between cadmium and the liver have been animal experiments [5-7]. The published human studies regarding liver damage $[8,9]$ mostly analyzed levels of gamma glutamyl transferase (GGT) and alkaline phosphatase (ALP). However, these are supplemental biomarkers in assessing liver damage; in fact, alanine aminotransferase (ALT) and aspartate aminotransferase (AST) have a higher diagnostic value in investigating liver injuries $[10,11]$. Some studies have reported no association between cadmium and aminotransferase levels [12-14], while others have confirmed associations in a specific population, but not the general population $[15,16]$. A few studies have reported relationships with serum cadmium; however, this parameter reflects short-term exposure to cadmium $[8,17,18]$. One study analyzed long-term cadmium exposure, but the serum liver function parameters were confined to ALT and GGT [19].

Thus, we aimed to investigate the relationship between cadmium and liver function parameters in the general population of the United States, where urinary cadmium, as an indicator of chronic exposure, was measured and 5 liver function parameters were used: ALT, AST, GGT, total bilirubin (TB), and ALP.

\section{METHODS}

\section{Study Population}

The National Health and Nutrition Examination Survey (NHANES), conducted by the National Center for Health Statistics (NCHS) of the Centers for Disease Control and Prevention, is a nationwide study that evaluates the health and nutritional status of the non-institutionalized civilian population of the United States.

The data used in this study were generated by combining 9 cycles of the survey between 1999 and 2016, including
NHANES 1999-2000, 2001-2002, 2003-2004, 2005-2006, 20072008, 2009-2010, 2011-2012, 2013-2014, and 2015-2016. From a total of 92062 participants in the pooled data, 49512 adults aged 20 years or older were selected for this study. Subsequently, we excluded individuals whose urinary cadmium data were missing ( $n=33$ 916). We further excluded participants whose data regarding all of the following liver function parameters were absent ( $\mathrm{n}=849)$ : ALT, AST, GGT, TB, and ALP. Finally, 1812 patients without data for covariates and 405 patients without data or weight were excluded, resulting in an eligible population of 12530 .

\section{Measurement of Urinary Cadmium Levels}

The NHANES provided a detailed laboratory procedure manual from urine sample collection to test principles [20]. In brief, inductively coupled plasma mass spectrometry, which is a multielement analytical technique, was used to measure the urinary cadmium concentration. The limits of detection (LODs) of cadmium were different across the study cycles: $0.060 \mu \mathrm{g} / \mathrm{L}$ from 1999 to $2004,0.042 \mu \mathrm{g} / \mathrm{L}$ from 2005 to 2012 , and $0.036 \mu \mathrm{g} / \mathrm{L}$ from 2013 to 2016. Regardless of the cycles, participants whose urinary cadmium levels were below the LOD $(n=687,5.5 \%$ of the eligible population) were not included in this study. For the first 2 cycles of the NHANES, urinary cadmium levels were adjusted using urinary molybdenum levels because molybdenumbased interference, which is inevitable consequence of inductively coupled plasma mass spectrometry, caused urinary cadmium levels to be overestimated [21]. If the adjusted levels were less than 0 , they were reported as 0 ; since this was not a meaningful result, we excluded participants whose values of urinary cadmium were $0(n=5)$ [22]. Finally, the study population $(n=11838)$ was determined.

\section{Liver Function Parameters}

The 5 analytes used to evaluate liver function were ALT, AST, GGT, TB, and ALP. The instrumentation used to measure liver function parameters differed from year to year: Hitachi Model 917 multichannel analyzer (Roche Diagnostics, Indianapolis, IN, USA) from 1999 to 2002, Beckman Synchron LX20 (Beckman Coulter, Brea, CA, USA) from 2003 to 2007, Beckman Uni$\mathrm{Cel}^{\mathbb{B}}$ DxC800 Synchron from 2008 to 2014, and Beckman Uni$\mathrm{Cel}^{\circledR}$ DxC 800 Synchron \& Beckman UniCel ${ }^{\circledR}$ DxC 660i Synchron Access Clinical Systems from 2015 to 2016. In the study population, there were 5 participants whose TB levels were reported as 0 , which was not a reportable value [23]. They were not ex- 
cluded during determining the study population because the values of their other liver function parameters were greater than 0 . However, they were excluded while exploring the relationship between cadmium and TB, as their TB values were invalid. Elevated levels of each parameter were defined as follows: ALT $>47 \mathrm{U} / \mathrm{L}$ in male and $>30 \mathrm{U} / \mathrm{L}$ in female, AST $>33 \mathrm{U} / \mathrm{L}$ in both sexes, GGT $>65 \mathrm{U} / \mathrm{L}$ in male and $>36 \mathrm{U} / \mathrm{L}$ in female, TB $>1.3 \mathrm{mg} / \mathrm{dL}$ in both sexes, and ALP $>113 \mathrm{U} / \mathrm{L}$ in both sexes [24]. However, the values for the first cycle were different and were as follows: ALT $>41 \mathrm{U} / \mathrm{L}$ in male and $>31 \mathrm{U} / \mathrm{L}$ in female, AST $\geq 37 \mathrm{U} / \mathrm{L}$ in male and $\geq 31 \mathrm{U} / \mathrm{L}$ in female, GGT $>49 \mathrm{U} / \mathrm{L}$ in male and $>32 \mathrm{U} / \mathrm{L}$ in female, $\mathrm{TB}>1.0 \mathrm{mg} / \mathrm{dL}$ in both sexes, and ALP $>117 \mathrm{U} / \mathrm{L}$ in both sexes [25].

\section{Other Variables of Interest}

Data regarding the following potential confounders were obtained through questionnaires and examination data from NHANES: age (as a continuous variable), sex (male or female), race/ethnicity (non-Hispanic White, non-Hispanic Black, Hispanic, or others), annual family income (less than US\$20 000 or US\$20 000 or more), smoking status (current smoker, former smoker, or never smoker), alcohol consumption status (drinker or non-drinker), and physical activity (yes or no). Body mass index (BMl; $\left.\mathrm{kg} / \mathrm{m}^{2}\right)$ was categorized into 4 classes: underweight $\left(<18.5 \mathrm{~kg} / \mathrm{m}^{2}\right)$, normal weight $\left(18.5-24.9 \mathrm{~kg} / \mathrm{m}^{2}\right)$, overweight $\left(25.0-29.9 \mathrm{~kg} / \mathrm{m}^{2}\right)$, and obese $\left(\geq 30.0 \mathrm{~kg} / \mathrm{m}^{2}\right)$. In addition, hepatitis $B$ virus (HBV) infection and hepatitis $C$ virus (HCV) infection were defined as seropositivity for hepatitis $B$ surface antigen and hepatitis C viral RNA, respectively. Heavy drinking was defined as drinking 5 or more drinks daily. However, between 2013 and 2016, the definition was slightly changed as having 5 or more drinks daily for male and having 4 or more drinks daily for female. Urinary creatinine levels, which were used to account for varying urine dilution, were measured using a Beckman Synchron CX3 Clinical Analyzer from 1999 to 2007, Roche/Hitachi Modular P Chemistry Analyzer from 2008 to 2012, and Roche Cobas 6000 Analyzer from 2013 to 2016.

\section{Statistical Analysis}

Urinary cadmium and liver function parameters were logtransformed to achieve normality. Weighted estimates were obtained according to the NHANES survey methods and analytic guidelines. Linear regression analyses were conducted to investigate the relationships between urinary cadmium levels and liver function parameters. Logistic regression analyses were also conducted to determine the odds ratios (ORs) and 95\% confidence intervals ( $\mathrm{Cls}$ ) of higher liver function parameters per unit increase in log-transformed urinary cadmium.

While performing both regression models, correcting for the dilution of urine with urinary creatinine was achieved in the following 2 ways: a creatinine-adjusted model and a volumebased model. In the creatinine-adjusted model, which is the traditional method to adjust for urine dilution, urinary cadmium divided by urinary creatinine was used to construct the regression model. However, as the urinary concentration of creatinine varies according to demographic features such as age, sex, and race/ethnicity, the creatinine-adjusted model is not recommended when the population is composed of individuals with diverse demographic characteristics [26]. To overcome this limitation, a new method called the volume-based model was devised, in which urinary creatinine is used as a covariate rather than a denominator [26].

In terms of confounders, 2 models were designed: model 1 was a crude model, which was not adjusted for any covariates, while model 2 was a fully adjusted model with covariates including age, sex, race/ethnicity, annual family income, smoking status, alcohol consumption status, physical activity, and BMI. In addition, 3 more models were fitted. Model 3 additionally included HBV infection as well as HCV infection and heavy drinking as covariates. Model 4 , as a sensitivity analysis, was performed in a similar fashion as the regression analysis in model 2, albeit with a new study population generated by excluding HBV-infected participants, HCV-infected participants, and heavy drinkers from the original study population. Model 5 , another sensitivity analysis, was performed along similar lines as in model 2 except for the fact that it excluded smokers and former smokers from the original study population. Four more sensitivity analyses were conducted as follows: (1) an interaction term between smoking and alcohol consumption was considered; (2) an interaction term between smoking and cadmium levels was considered; (3) an interaction term between alcohol consumption and cadmium levels was considered; and (4) the analysis was performed with participants aged 20 years to 59 years old.

All analyses were conducted with SAS version 9.4 (SAS Institute Inc., Cary, NC, USA) using the PROC SURVEYREG and PROC SURVEYLOGISTIC procedures. The level of statistical significance was set at $\alpha=0.05$. 


\section{Ethics Statement}

The NCHS Institutional Review Board (before 2003) and the NCHS Research Ethics Review Board (from 2003 to the present) approved the study protocols of the NHANES. All participants provided oral or written informed consent for the survey.

\section{RESULTS}

Table 1 presents the general characteristics of the study population. The mean age of the population was 50.12 years (standard deviation, 18.02), and females accounted for $50.2 \%$ of them. Most of the participants were non-Hispanic White (46.7\%), not lower-income (73.3\%), never smokers (52.2\%), and drinkers (70.4\%). Among drinkers, heavy drinkers accounted for $21.1 \%$. Only a few people were infected with HBV (0.4\%) and HCV (1.5\%).

Five subsamples were used to analyze the association between urinary cadmium and each liver function parameter (ALT, AST, GGT, TB, and ALP). Each subsample was generated by excluding the participants with missing data regarding the corresponding liver function parameter. The subsamples were different from each other because an individual with missing data on one liver function parameter differed from an individual with missing data on another liver function parameter. The subsample sizes and geometric means ( $95 \% \mathrm{Cls}$ ) of the corresponding liver function parameters, urinary cadmium, and creatinine-adjusted urinary cadmium (urinary cadmium divided by urinary creatinine) are presented in Table 2 . The sizes of the subsamples used to analyze the effect of cadmium on ALT, AST, GGT, TB, and ALP were 11 818, 11 818, 11 838, 10 556, and 10564 , respectively. The geometric means of ALT, AST, GGT, $\mathrm{TB}$, and ALP were $22.35 \mathrm{U} / \mathrm{L}, 23.95 \mathrm{U} / \mathrm{L}, 22.24 \mathrm{U} / \mathrm{L}, 0.63 \mathrm{mg} / \mathrm{dL}$, and $67.23 \mathrm{U} / \mathrm{L}$, respectively. The geometric means of urinary cadmium and creatinine-adjusted urinary cadmium were almost the same when compared across each subsample.

Table 3 summarizes the beta coefficients and standard errors (SEs) between the urinary cadmium levels and liver function parameters. The fully adjusted model (model 2 ) revealed that all the liver function parameters were positively associated with urinary cadmium, regardless of whether the volume-based or creatinine-adjusted model was selected. In the volume-based model, a 1-unit increase in the log-transformed urinary cadmium level was associated with increases of 0.049 (SE, 0.008; $p<0.001), 0.030$ (SE, 0.006; $p<0.001), 0.093$ (SE, 0.011; $p<0.001$ ), 0.034 (SE, 0.009; $p<0.001$ ), and 0.040 (SE, 0.005; $p<0.001$ ) in log-transformed ALT, AST, GGT, TB, and ALP levels, respectively.
Table 1. Characteristics of the study population

\begin{tabular}{|c|c|}
\hline Characteristics & Mean \pm SD or $\mathbf{n}(\%)$ \\
\hline No. of participants & 11838 \\
\hline Age (y) & $50.12 \pm 18.02$ \\
\hline \multicolumn{2}{|l|}{ Sex } \\
\hline Male & 5897 (49.8) \\
\hline Female & $5941(50.2)$ \\
\hline \multicolumn{2}{|l|}{ Race/ethnicity } \\
\hline Non-Hispanic White & 5532 (46.7) \\
\hline Non-Hispanic Black & $2375(20.1)$ \\
\hline Hispanic & 3063 (25.9) \\
\hline Others & $868(7.3)$ \\
\hline \multicolumn{2}{|c|}{ Annual family income (US\$) } \\
\hline$<20000$ & $3162(26.7)$ \\
\hline$\geq 20000$ & 8676 (73.3) \\
\hline \multicolumn{2}{|l|}{ Smoking status } \\
\hline Current smoker & $2500(21.1)$ \\
\hline Former smoker & $3154(26.6)$ \\
\hline Never smoker & $6184(52.2)$ \\
\hline \multicolumn{2}{|c|}{ Alcohol consumption status } \\
\hline Heavy drinker & $1755(14.8)$ \\
\hline Moderate drinker & $6581(55.6)$ \\
\hline Non-drinker & $3502(29.6)$ \\
\hline \multicolumn{2}{|l|}{ Physical activity } \\
\hline Yes & $5114(43.2)$ \\
\hline No & $6724(56.8)$ \\
\hline \multicolumn{2}{|l|}{ BMI } \\
\hline Underweight & $183(1.5)$ \\
\hline Normal weight & $3252(27.5)$ \\
\hline Overweight & 4134 (34.9) \\
\hline Obese & $4269(36.1)$ \\
\hline HBV infection & $52(0.4)$ \\
\hline HCV infection & $173(1.5)$ \\
\hline
\end{tabular}

BMI, body mass index; HBV, hepatitis B virus; HCV, hepatitis C virus; SD, standard deviation.

Additional adjustment for HBV infection, HCV infection, and heavy drinking (model 3 ) did not change the statistical significance of these findings, although the beta coefficient values decreased. Among the crude relationships, AST, GGT, and ALP levels were positively associated with urinary cadmium levels, with statistical significance; however, ALT and TB levels showed no significant relationships with urinary cadmium levels. A sensitivity analysis excluding those with HBV or HCV infection and heavy drinkers (model 4) showed that the significance remained unchanged when compared to model 2. The significance of the relationship was also maintained in another sen- 
Table 2. Mean values of the corresponding liver function parameters and urinary cadmium levels among the subjects included in each analysis

\begin{tabular}{|c|c|c|c|c|c|}
\hline Exposure or clinical variables & ALT $(n=11818)$ & AST $(n=11818)$ & GGT (n=11 838) & TB $(n=10556)$ & $\operatorname{ALP}(n=10564)$ \\
\hline $\begin{array}{l}\text { Corresponding liver function parameter } \\
\text { (U/L or } \mathrm{mg} / \mathrm{dL})\end{array}$ & $22.35(8.86,56.38)$ & $23.95(12.44,46.11)$ & $22.24(6.11,80.94)$ & $0.63(0.27,1.48)$ & $67.23(36.10,125.22)$ \\
\hline $\begin{array}{l}\text { Creatinine-adjusted urinary cadmium } \\
\text { ( } \mu \mathrm{g} / \mathrm{g} \text { creatinine })\end{array}$ & $0.27(0.05,1.39)$ & $0.27(0.05,1.39)$ & $0.27(0.05,1.40)$ & $0.27(0.05,1.37)$ & $0.27(0.05,1.37)$ \\
\hline
\end{tabular}

Values are presented as geometric mean (95\% confidence interval).

ALT, alanine aminotransferase; AST, aspartate aminotransferase; GGT, gamma glutamyl transferase; TB, total bilirubin; ALP, alkaline phosphatase.

Table 3. Linear regression analyses of log-transformed urinary cadmium and log-transformed liver function parameters

\begin{tabular}{|c|c|c|c|c|c|c|c|c|c|c|}
\hline Variables $^{1}$ & Model 1 & $p$-value & Model 2 & $p$-value & Model 3 & $p$-value & Model 4 & $p$-value & Model 5 & $p$-value \\
\hline $\operatorname{ALT}(n=11818)$ & $0.005 \pm 0.007$ & 0.435 & & & & & & & & \\
\hline Volume-based model & - & & $0.049 \pm 0.008$ & $<0.001$ & $0.042 \pm 0.008$ & $<0.001$ & $0.035 \pm 0.008$ & $<0.001$ & $0.059 \pm 0.010$ & $<0.001$ \\
\hline Creatinine-adjusted model & - & & $0.066 \pm 0.008$ & $<0.001$ & $0.056 \pm 0.008$ & $<0.001$ & $0.051 \pm 0.008$ & $<0.001$ & $0.074 \pm 0.010$ & $<0.001$ \\
\hline AST (n=11 818) & $0.020 \pm 0.005$ & $<0.001$ & & & & & & & & \\
\hline Volume-based model & - & & $0.030 \pm 0.006$ & $<0.001$ & $0.024 \pm 0.006$ & $<0.001$ & $0.017 \pm 0.006$ & 0.009 & $0.033 \pm 0.008$ & $<0.001$ \\
\hline Creatinine-adjusted model & - & & $0.045 \pm 0.007$ & $<0.001$ & $0.037 \pm 0.007$ & $<0.001$ & $0.029 \pm 0.007$ & $<0.001$ & $0.044 \pm 0.008$ & $<0.001$ \\
\hline GGT (n=11 838) & $0.096 \pm 0.008$ & $<0.001$ & & & & & & & & \\
\hline Volume-based model & - & & $0.093 \pm 0.011$ & $<0.001$ & $0.084 \pm 0.011$ & $<0.001$ & $0.070 \pm 0.011$ & $<0.001$ & $0.071 \pm 0.013$ & $<0.001$ \\
\hline Creatinine-adjusted model & - & & $0.113 \pm 0.012$ & $<0.001$ & $0.101 \pm 0.012$ & $<0.001$ & $0.086 \pm 0.012$ & $<0.001$ & $0.083 \pm 0.014$ & $<0.001$ \\
\hline $\mathrm{TB}(\mathrm{n}=10556)$ & $0.007 \pm 0.008$ & 0.367 & & & & & & & & \\
\hline Volume-based model & - & & $0.034 \pm 0.009$ & $<0.001$ & $0.034 \pm 0.009$ & $<0.001$ & $0.041 \pm 0.010$ & $<0.001$ & $0.080 \pm 0.013$ & $<0.001$ \\
\hline Creatinine-adjusted model & - & & $0.040 \pm 0.009$ & $<0.001$ & $0.040 \pm 0.009$ & $<0.001$ & $0.047 \pm 0.010$ & $<0.001$ & $0.085 \pm 0.013$ & $<0.001$ \\
\hline $\operatorname{ALP}(n=10564)$ & $0.045 \pm 0.004$ & $<0.001$ & & & & & & & & \\
\hline Volume-based model & - & & $0.040 \pm 0.005$ & $<0.001$ & $0.039 \pm 0.005$ & $<0.001$ & $0.037 \pm 0.006$ & $<0.001$ & $0.026 \pm 0.009$ & 0.004 \\
\hline Creatinine-adjusted model & - & & $0.049 \pm 0.006$ & $<0.001$ & $0.048 \pm 0.006$ & $<0.001$ & $0.047 \pm 0.007$ & $<0.001$ & $0.030 \pm 0.009$ & $<0.001$ \\
\hline
\end{tabular}

Values are presented as beta \pm standard error.

Model 1: Not adjusted for any covariates; Model 2: Adjusted for age, sex, race/ethnicity, annual family income, smoking status, alcohol consumption status, physical activity, and BMl; Model 3: Adjusted for age, sex, race/ethnicity, annual family income, smoking status, alcohol consumption status, physical activity, $\mathrm{BMI}, \mathrm{HBV}$ infection, HCV infection, and heavy drinking; Model 4: Excluded HBV-infected participants, HCV-infected participants, and heavy drinkers from the original study population and adjusted for age, sex, race/ethnicity, annual family income, smoking status, alcohol consumption status, physical activity, and BMl; Model 5: Excluded smokers and former smokers from the original study population and adjusted for age, sex, race/ethnicity, annual family income, smoking status, alcohol consumption status, physical activity, and BMI.

ALT, alanine aminotransferase; AST, aspartate aminotransferase; GGT, gamma glutamyl transferase; TB, total bilirubin; ALP, alkaline phosphatase; BMI, body mass index; HBV, hepatitis B virus; HCV, hepatitis C virus.

${ }^{1} T$ The sizes of subsamples used in models 1, 2, and 3 were indicated. The number of participants used in model 4 and 5 was different as follows: for model 4 , the numbers of participants used to analyze the effect of cadmium on ALT, AST, GGT, TB, and ALP were 9928, 9928, 9944, 8843, and 8847, respectively; for model 5 , the numbers of participants used to analyze the effect of cadmium on ALT, AST, GGT, TB, and ALP were 6174, 6174, 6184, 5545, and 5547, respectively.

sitivity analysis that was performed only with never-smokers after excluding current smokers and former smokers from the original study population (model 5).

Table 4 shows the ORs ( $95 \% \mathrm{Cls})$ of elevated liver function parameters per unit increase in the log-transformed urinary cadmium level. Both volume-based and creatinine-adjusted models showed that higher urinary cadmium levels were associated with increased liver function parameters after adjustment for potential confounders (model 2). When the volume- based model was used, the ORs ( $95 \% \mathrm{Cls}$ ) of having higher ALT, AST, GGT, TB, and ALP per unit increase in the log-transformed urinary cadmium level were 1.360 (1.210 to 1.528$), 1.307$ (1.149 to 1.486 ), 1.520 (1.357 to 1.704 ), 1.201 (1.003 to 1.438 ), and 1.568 (1.277 to 1.926). Even after adjusting for HBV infection, HCV infection, and heavy drinking (model 3), most of these relationships remained statistically significant; however, the relationship between TB and urinary cadmium became statistically non-significant when the volume-based model was ap- 
Table 4. Odds ratios $(95 \% \mathrm{Cls}$ ) of higher liver function parameters per 1-unit increase in the log-transformed urinary cadmium level

\begin{tabular}{|c|c|c|c|c|c|}
\hline Variables $^{1}$ & Model 1 & Model 2 & Model 3 & Model 4 & Model 5 \\
\hline $\operatorname{ALT}(n=11818)$ & $1.138(1.054,1.230)$ & & & & \\
\hline Volume-based model & & $1.360(1.210,1.528)$ & $1.310(1.163,1.477)$ & $1.242(1.090,1.415)$ & $1.401(1.181,1.662)$ \\
\hline AST (n=11 818) & $1.179(1.083,1.283)$ & & & & \\
\hline Volume-based model & & $1.307(1.149,1.486)$ & $1.254(1.096,1.435)$ & $1.211(1.042,1.408)$ & $1.299(1.095,1.540)$ \\
\hline Volume-based model & & $1.520(1.357,1.704)$ & $1.475(1.312,1.657)$ & 1.390 (1.209, 1.598) & $1.447(1.224,1.711)$ \\
\hline Creatinine-adjusted model & & $1.611(1.416,1.833)$ & $1.544(1.356,1.759)$ & $1.443(1.237,1.684)$ & $1.530(1.292,1.811)$ \\
\hline $\mathrm{TB}(\mathrm{n}=10556)$ & $1.044(0.921,1.183)$ & & & & \\
\hline Volume-based model & & $1.201(1.003,1.438)$ & $1.173(0.974,1.413)$ & $1.256(1.021,1.545)$ & $1.399(1.096,1.785)$ \\
\hline Creatinine-adjusted model & & $1.804(1.457,2.233)$ & $1.738(1.409,2.145)$ & $1.728(1.351,2.211)$ & $1.629(1.222,2.171)$ \\
\hline
\end{tabular}

Values are presented as odds ratio (95\% confidence interval).

Model 1: Not adjusted for any covariates; Model 2: Adjusted for age, sex, race/ethnicity, annual family income, smoking status, alcohol consumption status, physical activity, and BMl; Model 3: Adjusted for age, sex, race/ethnicity, annual family income, smoking status, alcohol consumption status, physical activity, BMI, HBV infection, HCV infection, and heavy drinking; Model 4: Excluded HBV-infected participants, HCV-infected participants, and heavy drinkers from the original study population and adjusted for age, sex, race/ethnicity, annual family income, smoking status, alcohol consumption status, physical activity, and BMI; Model 5: Excluded smokers and former smokers from the original study population and adjusted for age, sex, race/ethnicity, annual family income, smoking status, alcohol consumption status, physical activity, and BMI.

ALT, alanine aminotransferase; AST, aspartate aminotransferase; GGT, gamma glutamyl transferase; TB, total bilirubin; ALP, alkaline phosphatase; BMI, body mass index; HBV, hepatitis B virus; HCV, hepatitis C virus.

${ }^{1}$ The sizes of subsamples used in models 1, 2, and 3 were indicated. The number of participants used in model 4 and 5 was different as follows: for model 4 , the numbers of participants used to analyze the effect of cadmium on ALT, AST, GGT, TB, and ALP were 9928, 9928, 9944, 8843, and 8847, respectively; for model 5, the numbers of participants used to analyze the effect of cadmium on ALT, AST, GGT, TB, and ALP were 6174,6174, 6184, 5545, and 5547, respectively.

plied. Non-significance of the relationship between urinary cadmium and TB was also observed in the crude model (model 1). The results of both sensitivity analyses (models 4 and 5 ) were significant as in model 2 .

Supplemental Materials 1-3 show the results of sensitivity analyses considering smoking and alcohol consumption, smoking and cadmium levels, and alcohol consumption and cadmium levels, respectively. Interestingly, most of the significant associations were not compromised by the various interaction terms considered in this study. The results of the sensitivity analysis conducted with participants aged 20 years to 59 years old are presented in Supplemental Material 4; most of the relationships remained significant except for those with TB levels.

\section{DISCUSSION}

In the general population of the United States, urinary levels of cadmium were found to be positively associated with the following liver function parameters: ALT, AST, GGT, TB, and ALP. Excluding and adjusting for HBV infection, HCV infection, and heavy drinking did not affect most of the relationships, which showed significant positive estimates. In particular, significant relationships were observed among non-smokers, suggesting that cadmium is a risk factor independent of smoking.

To the best of our knowledge, this is the first study to investigate the effect of cadmium on ALT, AST, GGT, TB, and ALP levels simultaneously. Furthermore, the relationships between urinary cadmium and AST or TB in the general population have been reported for the first time. However, some previous studies have shown a significant association between cadmium exposure and hepatotoxicity under one of the following conditions: (1) liver injury was assessed by some of the abovementioned liver function parameters; (2) serum cadmium was measured instead of urinary cadmium; and (3) the associations were investigated in special populations, not in the general population. Previous studies using data from the NHANES III from 1988 
to 1994 reported that higher urinary cadmium levels were related to elevated serum ALT or GGT levels $[9,19]$. Furthermore, some studies conducted using the NHANES data showed that the increased urinary cadmium levels were positively associated with serum GGT or ALP levels $[8,27,28]$. Several previous studies measured serum cadmium levels to assess cadmium exposure; however, serum cadmium only reflects acute exposure $[16-18,28]$. Given that the half-life of urinary cadmium is immensely long ( 16 years in male and 20 years in female), it is more appropriate than serum cadmium for evaluating the effect of cadmium on the human body [29]. A few other studies were performed in special populations, such as patients on chronic peritoneal dialysis [16], non-smoking female in rural areas [30], tobacco workers [31], and patients in a coronary care unit [15]. Despite these limitations, the results of the aforementioned studies have mostly revealed that cadmium is related to liver injury, which is consistent with our results.

However, the findings of a few studies were inconsistent with the results of the present study, in that there was no relationship between serum cadmium and ALT levels; all the incompatible results were derived using the same data, NHANES 2003-2004 [12-14]. This disparity can be explained in 2 ways. First, although it is known that acute exposure to cadmium causes liver injury [7] and serum cadmium is appropriate for diagnosing short-term exposure to cadmium [9,32], it is not an appropriate marker for evaluating disease severity [32] because the relatively long half-life of serum cadmium (75 to 128 days) could show false positivity [33]. Second, the proportion of data below the LOD regarding blood cadmium was $8.8 \%$ in the NHANES 2003-2004 cycle. A considerable segment of the population could have a wide spectrum of values; however, they were categorized as 1 group, such as the reference group or first quartile. Inadequate handling of heterogeneity may have caused residual confounding. Considering that our results were consistent with the results of some population-based studies, which revealed an association between urinary cadmium levels and liver damage $[9,10,20,28]$, a hypothesis can be suggested that chronic exposure to cadmium could cause hepatotoxicity. This hypothesis is supported by multiple animal studies. A recent study with 3-week-old Kunming mice revealed that chronic low-dose exposure to cadmium for 30 days caused elevation of both AST and ALT levels, infiltration of inflammatory cells in the liver, and upregulation of mRNA encoding proinflammatory and anti-inflammatory cytokines [5]. Another study with 3-week-old C57BL/6 mice reported abnormal his- topathological changes in the liver, such as liver fibrosis, infiltration of immune cells, and hepatic stellate cell activation, following environmental-level chronic oral administration of cadmium for 32 weeks [6].

Although the mechanism of hepatotoxicity due to cadmium still needs to be elucidated, the inflammatory response and oxidative stress are considered plausible explanations for this phenomenon. Cadmium causes the infiltration of polymorphonuclear neutrophils and Kupffer cells into the liver [34]. Cytokines produced by Kupffer cells, such as tumor necrosis factor-alpha, interleukin-1, and interleukin-6, have been shown to be associated with inflammation and subsequent hepatotoxicity [35]. The role of Kupffer cells in hepatotoxicity during cadmium exposure was demonstrated in a counterfactual situation, which showed that when Kupffer cells were suppressed, cadmium-induced hepatotoxicity was restricted [36]. Alternatively, an imbalance in redox homeostasis could be another reasonable hypothesis. In the human body, cadmium tends to bind to the sulfhydryl group, and the representative compounds containing the sulfhydryl group are glutathione and metallothionein. Metallothionein acts as a buffer against cadmium, forming a cadmium-metallothionein complex that could prevent cadmium-induced toxicity [37]. This indicates that either excess cadmium or deficiency of metallothionein could exacerbate the damage caused by cadmium. A study in mice showed that cadmium-induced liver injury was more severe in metallothionein-knockout mice than in normal mice [38]. Unbound cadmium promotes the activity of pro-oxidants and suppresses the level of antioxidants, resulting in oxidative stress. A study using rat liver cells found that exposure to cadmium was followed by an increased concentration of malondialdehyde, a marker of lipid peroxidation, and a decrease in the activity of antioxidant enzymes, such as superoxide dismutase, catalase, glutathione reductase, and glutathione peroxidase [39]. Because it is not clear how cadmium damages the liver, further study is needed to identify the mechanism of cadmium-induced hepatotoxicity.

This study has several limitations. First, as the data used in this study were cross-sectional, the results of this study do not warrant inferences regarding causal relationships. Moreover, as the liver is involved in the metabolism of cadmium, there is a possibility of reverse causality. However, this is relatively unlikely. Metallothionein, which induces cadmium retention, is mainly synthesized in the liver [40]. When the liver is damaged, metallothionein production is hampered, resulting in increased 
excretion of cadmium. Therefore, liver damage might result in a decreased cadmium concentration. However, as there could be numerous unknown mechanisms regarding cadmium metabolism, the possibility of reverse causality could not be completely excluded. Second, some unmeasured covariates could have created a spurious relationship. Study participants residing in cadmium-polluted areas or occupationally exposed workers might have had liver damage due to an unobserved confounder that was related to cadmium exposure. For example, acetaminophen overdose as a result of headache caused by cadmium exposure may have contributed in part to liver damage. However, this possibility could not be accounted for in the present study due to the lack of data. Third, the adjustment may not have been adequate because smoking, which affects cadmium concentrations, typically correlated well with alcohol consumption, which affects liver damage. Furthermore, smoking and alcohol consumption may affect the cadmium accumulation rate. However, the correlation between alcohol consumption and smoking was weak (Pearson correlation coefficient, $0.222 ; p<0.001)$. Moreover, most of the associations remained significant even when considering interactions between the variables (Supplemental Materials 1-3). Thus, it can be said that the significance of the relationships was confirmed again even using a more nuanced model. Similarly, age adjustment might have been insufficient in this study. As the elderly population tends to have compromised liver function as well as a higher cadmium concentration, which is attributed to its long half-life, their data reflecting prolonged accumulation might complicate the interpretation of the statistical analyses. Therefore, a sensitivity analysis in a younger population could provide insights by excluding this very-long-term cumulative effect. As most of the significant results remained unchanged in the sensitivity analysis of the younger population, it can be suggested that the main result of this study was not spurious due to age-associated factors. Finally, as the instrumentation and questionnaire varied during the 18 years of study, there could have been measurement bias. For example, the LODs of urinary cadmium were different from cycle to cycle. However, to minimize this source of error, urinary cadmium data below the LOD were excluded rather than being imputed as a constant value, such as the LOD divided by the square root of 2 . Otherwise, the value of $L O D / \sqrt{ } 2$ in earlier studies could have been greater than some detected concentrations of urinary cadmium in later studies because the LODs of urinary cadmium decreased from 1999 to 2015.
In conclusion, we found that ALT, AST, GGT, TB, and ALP levels were positively associated with urinary cadmium concentrations in the United States. This implies that chronic cadmium exposure may cause hepatotoxicity in humans. However, as causality cannot be proven via cross-sectional studies, longitudinal studies such as cohort studies should be performed to investigate whether such a causal relationship exists.

\section{SUPPLEMENTAL MATERIALS}

Supplemental materials are available at https://doi.org/10. 3961/jpmph.21.435.

\section{CONFLICT OF INTEREST}

The authors have no conflicts of interest associated with the material presented in this paper.

\section{FUNDING}

This work was supported by the Basic Science Research Program through the National Research Foundation of Korea (NRF) funded by the Ministry of Education, Science and Technology (grant No. 2019R1A2C1004966 and 2020R1A2C1102097). This research was also funded by the Education and Research Encouragement Fund of Seoul National University Hospital.

\section{ACKNOWLEDGEMENTS}

None.

\section{AUTHOR CONTRIBUTIONS}

Conceptualization: DH, Data curation: JYM, KBM. Formal analysis: DH, JYM, KBM. Funding acquisition: KBM. Methodology: $\mathrm{DH}$, JYM, KBM. Project administration: KBM. Visualization: $\mathrm{DH}$, JYM. Writing - original draft: DH. Writing - review \& editing: $\mathrm{DH}, \mathrm{JYM}, \mathrm{KBM}$.

\section{ORCID}

Dongui Hong https://orcid.org/0000-0002-6418-3796

Jin-Young Min https://orcid.org/0000-0002-6578-7348

Kyoung-Bok Min https://orcid.org/0000-0001-9576-0093 


\section{REFERENCES}

1. Jacobo-Estrada T, Santoyo-Sánchez M, Thévenod F, Barbier O. Cadmium handling, toxicity and molecular targets involved during pregnancy: lessons from experimental models. Int J Mol Sci 2017;18(7):1590.

2. Korean Society for Preventive Medicine. Preventive medicine and public health. 3rd ed. Seoul: Gyechuk Munwhasa; 2019, p. 756-757 (Korean).

3. Genchi G, Sinicropi MS, Lauria G, Carocci A, Catalano A. The effects of cadmium toxicity. Int J Environ Res Public Health 2020; 17(11):3782.

4. Branca JJV, Morucci G, Pacini A. Cadmium-induced neurotoxicity: still much ado. Neural Regen Res 2018;13(11):1879-1882.

5. Li X, Li H, Cai D, Li P, Jin J, Jiang X, et al. Chronic oral exposure to cadmium causes liver inflammation by NLRP3 inflammasome activation in pubertal mice. Food Chem Toxicol 2021;148: 111944.

6. Xu Y, Mu W, Li J, Ba Q, Wang H. Chronic cadmium exposure at environmental-relevant level accelerates the development of hepatotoxicity to hepatocarcinogenesis. Sci Total Environ 2021; 783:146958.

7. Zou H, Wang T, Yuan J, Sun J, Yuan Y, Gu J, et al. Cadmium-induced cytotoxicity in mouse liver cells is associated with the disruption of autophagic flux via inhibiting the fusion of autophagosomes and lysosomes. Toxicol Lett 2020;321:32-43.

8. Colacino JA, Arthur AE, Ferguson KK, Rozek LS. Dietary antioxidant and anti-inflammatory intake modifies the effect of cadmium exposure on markers of systemic inflammation and oxidative stress. Environ Res 2014;131:6-12.

9. Lee DH, Lim JS, Song K, Boo Y, Jacobs DR Jr. Graded associations of blood lead and urinary cadmium concentrations with oxidative-stress-related markers in the U.S. population: results from the third National Health and Nutrition Examination Survey. Environ Health Perspect 2006;114(3):350-354.

10. Dufour DR, Lott JA, Nolte FS, Gretch DR, Koff RS, Seeff LB. Diagnosis and monitoring of hepatic injury. I. Performance characteristics of laboratory tests. Clin Chem 2000;46(12):20272049.

11. Ozer J, Ratner M, Shaw M, Bailey W, Schomaker S. The current state of serum biomarkers of hepatotoxicity. Toxicology 2008; 245(3):194-205.

12. Wahlang B, Appana S, Falkner KC, McClain CJ, Brock G, Cave $M C$. Insecticide and metal exposures are associated with a surrogate biomarker for non-alcoholic fatty liver disease in the National Health and Nutrition Examination Survey 20032004. Environ Sci Pollut Res Int 2020;27(6):6476-6487.

13. Yorita Christensen KL, Carrico CK, Sanyal AJ, Gennings C. Multiple classes of environmental chemicals are associated with liver disease: NHANES 2003-2004. Int J Hyg Environ Health 2013;216(6):703-709.

14. Cave M, Appana S, Patel M, Falkner KC, McClain CJ, Brock G. Polychlorinated biphenyls, lead, and mercury are associated with liver disease in American adults: NHANES 2003-2004. Environ Health Perspect 2010;118(12):1735-1742.

15. Hsu CW, Weng CH, Lin-Tan DT, Chu PH, Yen TH, Chen KH, et al. Association of urinary cadmium with mortality in patients at a coronary care unit. PLoS One 2016;11(1):e0146173.

16. Hsu CW, Lin JL, Lin-Tan DT, Huang WH, Chen KH, Yen TH. Association between blood cadmium levels and malnutrition in peritoneal dialysis. BMC Nephrol 2014;15:17.

17. Kang MY, Cho SH, Lim YH, Seo JC, Hong YC. Effects of environmental cadmium exposure on liver function in adults. Occup Environ Med 2013;70(4):268-273.

18. Park E, Kim J, Kim B, Park EY. Association between environmental exposure to cadmium and risk of suspected non-alcoholic fatty liver disease. Chemosphere 2021;266:128947.

19. Hyder O, Chung M, Cosgrove D, Herman JM, Li Z, Firoozmand $A$, et al. Cadmium exposure and liver disease among US adults. J Gastrointest Surg 2013;17(7):1265-1273.

20. Centers for Disease Control and Prevention (CDC). Laboratory procedure manual: antimony, arsenic, barium, beryllium, cadmium, cesium, cobalt, lead, manganese, molybdenum, platinum, strontium, thallium, tin, tungsten, and uranium [cited 2021 Jun 28]. Available from: https://wwwn.cdc.gov/nchs/ data/nhanes/2015-2016/labmethods/UTAS_UTASS_UM_UMS_ I_MET.pdf.

21. Jarrett JM, Xiao G, Caldwell KL, Henahan D, Shakirova G, Jones RL. Eliminating molybdenum oxide interference in urine cadmium biomonitoring using ICP-DRC-MS. J Anal At Spectrom 2008;23(7):962-967.

22. Centers for Disease Control and Prevention (CDC). National Health and Nutrition Examination Survey, 2001-2002 data documentation, codebook, and frequencies; 2005 [cited 2021 Oct 13]. Available from: https://wwwn.cdc.gov/Nchs/Nhanes/ 2001-2002/L06HM_B.htm.

23. Centers for Disease Control and Prevention (CDC). Laboratory procedure manual: bilirubin [cited 2021 Oct 13]. Available from: https://wwwn.cdc.gov/nchs/data/nhanes/2015-2016/ labmethods/BIOPRO_I_MET_BILIRUBIN_DXC800and660i.pdf. 
24. Centers for Disease Control and Prevention (CDC). Biochemistry profile in refrigerated serum -- NHANES 2001-2002 [cited 2021 Jun 28]. Available from: https://wwwn.cdc.gov/nchs/ data/nhanes/2001-2002/labmethods/I18_b_met_biochemistry_profile.pdf.

25. Centers for Disease Control and Prevention (CDC). Biochemistry profile in refrigerated serum NHANES 1999-2000 [cited 2021 Jun 28]. Available from: https://wwwn.cdc.gov/nchs/ data/nhanes/1999-2000/labmethods/lab18_met_biochemistry_profile.pdf.

26. Barr DB, Wilder LC, Caudill SP, Gonzalez AJ, Needham LL, Pirkle $J$. Urinary creatinine concentrations in the U.S. population: implications for urinary biologic monitoring measurements. Environ Health Perspect 2005;113(2):192-200.

27. Obeng-Gyasi E. Chronic cadmium exposure and cardiovascular disease in adults. J Environ Sci Health A Tox Hazard Subst Environ Eng 2020;55(6):726-729.

28. Cheung BM, Ong KL, Wong LY. Elevated serum alkaline phosphatase and peripheral arterial disease in the United States National Health and Nutrition Examination Survey 1999-2004. Int J Cardiol 2009;135(2):156-161.

29. Ishizaki M, Suwazono Y, Kido T, Nishijo M, Honda R, Kobayashi $\mathrm{E}$, et al. Estimation of biological half-life of urinary cadmium in inhabitants after cessation of environmental cadmium pollution using a mixed linear model. Food Addit Contam Part A Chem Anal Control Expo Risk Assess 2015;32(8):1273-1276.

30. Zhang YR, Wang P, Liang XX, Tan CS, Tan JB, Wang J, et al. Associations between urinary excretion of cadmium and renal biomarkers in nonsmoking females: a cross-sectional study in rural areas of South China. Int J Environ Res Public Health 2015; 12(10):11988-12001.

31. Sişman AR, Bülbül M, Coker C, Onvural B. Cadmium exposure in tobacco workers: possible renal effects. J Trace Elem Med Biol 2003;17(1):51-55.

32. Hung YM, Chung HM. Acute self-poisoning by ingestion of cadmium and barium. Nephrol Dial Transplant 2004;19(5): 1308-1309.

33. Bernhoft RA. Cadmium toxicity and treatment. ScientificWorldJournal 2013;2013:394652.

34. Arroyo VS, Flores KM, Ortiz LB, Gómez-Quiroz LE, Gutiérrez-Ruiz MC. Liver and cadmium toxicity. J Drug Metab Toxicol 2012; S5:001.

35. Yamano T, DeCicco LA, Rikans LE. Attenuation of cadmium-induced liver injury in senescent male fischer 344 rats: role of Kupffer cells and inflammatory cytokines. Toxicol Appl Pharmacol 2000;162(1):68-75.

36. Sauer JM, Waalkes MP, Hooser SB, Kuester RK, McQueen CA, Sipes IG. Suppression of Kupffer cell function prevents cadmium induced hepatocellular necrosis in the male Sprague-Dawley rat. Toxicology 1997;121(2):155-164.

37. Rikans LE, Yamano T. Mechanisms of cadmium-mediated acute hepatotoxicity. J Biochem Mol Toxicol 2000;14(2):110-117.

38. Habeebu SS, Liu J, Liu Y, Klaassen CD. Metallothionein-null mice are more sensitive than wild-type mice to liver injury induced by repeated exposure to cadmium. Toxicol Sci 2000;55(1):223232.

39. Ikediobi CO, Badisa VL, Ayuk-Takem LT, Latinwo LM, West J. Response of antioxidant enzymes and redox metabolites to cadmium-induced oxidative stress in CRL-1439 normal rat liver cells. Int J Mol Med 2004;14(1):87-92.

40. Klaassen CD, Liu J, Diwan BA. Metallothionein protection of cadmium toxicity. Toxicol Appl Pharmacol 2009;238(3):215220. 